\title{
Kernos
}

Revue internationale et pluridisciplinaire de religion grecque antique

15 | 2002

Varia

\section{Sur les traces de la piété des Grecs : vivre, dire et penser}

\section{André Motte}

\section{(2) OpenEdition \\ Journals}

\section{Édition électronique}

URL : http://journals.openedition.org/kernos/1411

DOI : 10.4000/kernos. 1411

ISSN : 2034-7871

\section{Éditeur}

Centre international d'étude de la religion grecque antique

\section{Édition imprimée}

Date de publication : 1 janvier 2002

ISSN : 0776-3824

\section{Référence électronique}

André Motte, «Sur les traces de la piété des Grecs : vivre, dire et penser », Kernos [En ligne], 15 | 2002, mis en ligne le 22 avril 2011, consulté le 21 septembre 2020. URL : http://journals.openedition.org/ kernos/1411 ; DOI : https://doi.org/10.4000/kernos.1411 
Kernos, 15 (2002), p. 489-552.

\title{
Revue des Livres
}

\section{Compte rendu critique}

\author{
Sur les traces de la piété des Grecs : vivre, dire et penser ${ }^{1}$
}

\author{
par André MotTe
}

Étudier la piété des Grecs dans ses manifestations cultuelles tout d'abord (p. 19-84), puis dans le vocabulaire qui l'exprime et l'érige progressivement en une notion distincte (p. 85-172), dans la réflexion enfin dont elle a fait l'objet chez certains philosophes (p. 173-209), tel est, esquissé dans ses trois principales articulations, le programme ambitieux et attrayant que poursuit l'ouvrage. L'étude vient à son heure; comme l'observe justement l'A. dans un substantiel "prologue » (p. 9-16), les travaux nombreux et très diversifiés qui, depuis un demisiècle, ont été consacrés la religion grecque n'ont guère abordé la question de la piété, si ce n'est de façon incidente et partielle. Aucune étude d'importance et ciblant le thème dans son ensemble n'est parue depuis l'ouvrage de Johanna Bolkenstein (Hosios en Eusebês, Amsterdam, 1936), qui se limitait d'ailleurs à une enquête terminologique et dont certaines conclusions avaient grand besoin d'être révisées. Or, la piété n'est pas une donnée quelconque, mais tout à fait centrale quand on cherche à comprendre une religion, - à tout le moins une religion telle que celle-là, - dans ce qui la spécifie, dans ses ressorts profonds et dans les módalités les plus typiques de son fonctionnement. Pareille approche conduit, en effet, à prendre en compte à la fois la dimension objective et subjective du phénomène, à explorer l'expérience religieuse dans son vécu quotidien ( «comment les Grecs vivaient-ils leur religion ? », p. 9) et à sonder les relations que ce peuple entretenait avec les puissances surnaturelles dont il appréhendait constamment la présence à ses côtés. C'est donc s'interroger sur le sens de leur comportement religieux. Ce n'est pas là, chacun en conviendra, une entreprise aisée, d'autant que la religion imprègne la vie et la pensée des Anciens dans la plupart de leurs modalités d'expression et que le complexe formé par celles-ci n'est pas resté figé durant les nombreux siècles que compte leur histoire. Il faut donc savoir gré à l'A. d'avoir tenté cet essai périlleux et, pour le dire tout de suite, de l'avoir fait avec perspicacité et finesse, dans une grande cohérence aussi, en dépit de la diversité des points de vue successivement abordés. Cette cohérence tient à la nature même du projet, clairement annoncé et expliqué : enquêter sur « les formes et les valeurs de la piété dans la Grèce des cités, dans son contexte historique, social et culturel »(p.13), ce qui ne prend sens qu'en référence

1 À propos de Louise Bruit ZaIdman, Le commerce des dieux. Eusebeia, essai sur la piété en Grèce ancienne, Paris, Éditions La Découverte, 2000. 1 vol. 13,5×22 cm, 240 p., 17 fig. (Textes à l'appui. Série bistoire classique). ISBN : 2-7071-3258-6. 
constante au cadre de la polis. Ce sont des documents, - littéraires, épigraphiques et iconographique, - puisés aux époques archaïque et classique qui fourniront principalement la matière première de l'étude, mais bien des observations glanées ne seraient pas infirmées aux époques ultérieures, car d'Homère aux début de l'ère nouvelle, d'importantes continuités se manifestent. À plusieurs reprises, l'A. mettra en garde contre une tendance à juger de la piété grecque à la lumière de la religion chrétienne qui nous est plus familière, mais qui se fonde sur de tout autres bases, à savoir l'idéal d'une rencontre intime avec un Dieu unique et personnel. On ne peut que souscrire à cette mise en garde, non sans observer qu'il est d'autres conceptions et schèmes d'interprétation modernes susceptibles de faire violence à la réalité antique pour peu qu'on les lui applique sans discernement.

La première partie de l'ouvrage témoigne très particulièrement du dessein global défini dans le prologue. On y passe en revue, en effet, les rites, les pratiques, les comportements, bref les principales manifestations, tant publiques que privées, de la piété grecque, cette notion ayant été au préalable esquissée, par provision, pourrait-on dire, en attendant l'enquête sur les mots. Il est d'abord question des fêtes nombreuses qui valorisent certains espaces de la cité et qui suspendent le temps civique, illustrant de façon privilégiée l'intrusion du sacré dans la vie des communautés et offrant souvent à la piété des citoyens l'occasion de se donner en spectacle. Viennent les sacrifices et les offrandes, les libations et les prières qui ont pour cadre ces cérémonies festives, mais qui trouvent place aussi en bien d'autres circonstances; il est fait droit notamment à la variété particulière d'offrandes qu'on appelle les trapezômata, et encore aux dédicaces et aux offrandes votives qui entendent perpétuer le souvenir du geste pieux et attirer à l'avenir la générosité divine. Plusieurs pages bienvenues sont réservées au portrait-modèle de l'homme pieux (theosebês) qu'incarne le personnage de Chrémyle dans le Ploutos d'Aristophane car, par delà la parodie, c'est une sorte de «manuel de piété quotidienne » qu'esquisse cette comédie. Divination et oracles, restés florissants durant toute l'Antiquité, font l'objet d'un chapitre particulier; c'est qu'ils sont, à un titre spécial, révélateurs d'une mentalité religieuse et d'une dévotion pieuse à l'égard de divinités censées intervenir dans la vie des individus, comme aussi des collectivités civiques ou autres; il est intéressant d'observer ici la rivalité qui, en régime démocratique, peut naître entre la décision politique et la consultation d'un oracle ou d'un devin, particulièrement dans les situations de guerre. Un dernier chapitre est consacré aux mystères et aux initiations, célébrations qui manifestent une piété davantage chargée d'émotion et où s'exprime l'aspiration à un bonheur que peut procurer la rencontre avec le divin, ici-bas ou même après la mort; ces dévotions ne sont pas pour autant incompatibles avec une piété civique, même si certains groupes privés et secrets ont pu inspirer quelquefois la méfiance des cités, voire leur désaveu, comme le montre par exemple la controverse célèbre qui a opposé Démosthène à Eschine.

L'originalité et l'intérêt de cette section très réussie ne sont certes pas à rechercher dans la revue de ces diverses rubriques que ne néglige aucun bon manuel de religion grecque, mais bien dans la manière concrète et très vivante dont sont exploités des documents variés, judicieusement choisis, de manière à faire voir les mille et une facettes de cette piété qu'à l'intérieur de leurs petites cités les Grecs n'ont cessé d'activer en l'honneur de leurs dieux. Le tableau, assurément, ne se veut pas complet. Il eût été intéressant, par exemple, de dire un mot des voyages fréquents, parfois longs et souvent accomplis dans des conditions pénibles, qu'entreprenaient les Hellènes, individuellement ou en groupes, pour consulter tel oracle, recevoir telle initiation, solliciter une guérison, parti- 
ciper aux jeux; on a découvert, dans les sanctuaires, des offrandes de pieds dont certaines ont été interprétées comme étant des " pieds de pèlerins », témoins des pieux efforts endurés par ces intrépides marcheurs. C'eût été l'occasion de souligner aussi la dimension panhellénique que pouvait prendre parfois la piété grecque. Mais, dans ce genre de synthèse, on est acculé à faire des choix.

A peine plus austère, tant la plume reste alerte et les contextes relevés évocateurs, est la partie centrale de l'ouvrage vouée à l'étude de la notion de piété. L'A. en esquisse rapidement l'histoire, mettant celle-ci en parallèle avec l'évolution des modèles culturels et sociaux. Le monde aristocratique d'Homère, qui dépeint le divin à son image, atteste déjà l'ensemble des pratiques qu'impliquera l'idée de piété, même si le mot eusebeia lui-même n'est pas encore présent. Dans le premier chant de l'Iliade qui raconte la colère d'Achille, c'est surtout négativement, par l'outrage qu'Agamemnon fait subir au prêtre d'Apollon, que cette idée est illustrée; l'impiété du chef des Achéens apparaît comme une transgression des égards dus au dieu, et elle menace la survie de toute la communauté. Si eusebeia et les termes apparentés ne sont pas encore usités par l'aède, les formes simples sebomai et sebas à tout le moins le sont, de même que les verbes bazomai et aideistbai ainsi que le substantif aidôs ${ }^{1}$. Par ces mots peuvent être exprimés précisément les sentiments de crainte et de respect qu'inspirent les dieux ou certaines manifestations du sacré. Chez Hésiode, la notion de piété s'exprime surtout par l'idée de justice, Dikè étant fille de Zeus, père des dieux et des hommes. Le poète renouvelle la conception homérique de la justice qu'il oppose à l'bubris; au nom de l'ordre souverain du Cronide, il prend la défense des paysans que briment les sentences torses des seigneurs locaux. À l'inverse, c'est la protestation d'un aristocrate irrité par les revendications de ses adversaires que fait entendre Théognis lorsqu'il brandit l'étendart de la justice, étroitement associée à l'idée de piété que désignent explicitement, et pour la première fois, les mots eusebeia, eusebês et eusebein ainsi que bosios. Cette association, qui perdurera tout au long de la tradition classique, est typique de la dimension citoyenne qui marque la piété grecque : le respect des dieux et la loyauté entre les hommes ne sont pas séparables. Pindare voit dans l'eusebeia la qualité de l'homme qui s'acquitte avec exactitude de ses devoirs religieux. Tout un chapitre est ensuite réservé à un choix de lectures tragiques de la piété. Eschyle y tient la vedette, avec la trilogie de l'Orestie qui introduit au cœur de la cité le débat sur la justice et la piété, avec les Sept contre Thèbes aussi, qui fait la part entre une piété masculine et une piété féminine, toutes deux rapportées cependant à l'ordre civique. Font aussi une rapide apparition le Penthée des Bacchantes et l'infortuné Hippolyte, l'un et l'autre châtiés pour une forme exemplaire, mais bien différente, d'impiété; n'est pas oubliée non plus la petite Antigone qui s'oppose à Créon au nom de la piété que l'on sait. Suit une galerie de portraits tirés cette fois de l'histoire et opposant d'abord, à partir des relations de Thucydide et surtout de Plutarque, l'homme de guerre Nicias dont la piété réputée, et fort ostentatoire, tend à se confondre avec sa qualité d'homme public, et l'ambitieux Alcibiade dont l'impiété ne fut pas moins notoire, mais qui réussit à se faire réhabiliter par un acte éclatant de pieuse bravoure : par temps de guerre, il rendit possible le

1 Qu'on me permette de signaler ici un article remarquable, et tout récent, de J. Rudhardt sur ce sujet: "Quelques remarques sur la notion d'aidôs", in Kînot. De la religion à la pbilosopbie. Mélanges offerts à A. Motte, Liège, C.I.E.R.G.A., 2001 (Kernos, suppl. 11), p. 1-21; cette étude, qui porte essentiellement sur Homère et Eschyle, s'inspire d'un ouvrage dont Rudhardt vante les mérites et qu'on ne voit pas cité dans la bibliographie de Mme Bruit Zaidman : D.L. CaIrns, Aidôs. The Psychology and Etbics of Honour and Shame in Ancient Greek Literature, Oxford, 1993. 
pèlerinage pédestre vers Éleusis en plaçant le cortège sous la protection de son armée. Pour ces deux personnages, la piété était un objet de compétition sous le regard des autres. Moins ambiguë est sans doute la piété bien connue de Xénophon, qu'il ne faut pas juger, insiste l'A., à l'aune de la "spiritualité » de Socrate ou de Platon, ni davantage en fonction de critères qui appartiennent à notre propre culture, mais bien en suivant les regards portés sur lui par ses contemporains. On découvre alors que cette piété s'inscrit parmi les vertus sociales dûment reconnues et qu'elle englobe le respect des parents ainsi que l'ensemble de la cité. Ce qui n'a d'ailleurs pas empêché Xénophon de faire lui-même l'éloge de la piété de Socrate qu'il semble prendre en modèle et dont le moins qu'on puisse dire est qu'elle n'était pas calquée sur un modèle commun; il est donc mal venu, conclut l'A., de tenir pour fade et formelle la piété de l'auteur des Mémorables. Place est faite aussi, comme il se devait, au premier traité conservé Peri bosiou, à savoir l'Eutbyphron de Platon, dialogue qui trace un portrait peu flatteur d'un devin qui se prétend expert en choses religieuses et où Socrate s'efforce en vain de trouver avec lui une définition adéquate de la piété. C'est l'occasion pour l'A. d'examiner l'emploi concurrent, mais nettement moins abondant, que fait cette ouvre des mots de la famille d'eusebeia. Ceux-ci paraissent renvoyer davantage à une notion juridique de la piété, telle qu'elle était entrée dans l'usage à la faveur des procès d'impiété; là est peut-être une raison pour laquelle Platon lui préfère nettement les connotations que véhiculent les mots de la famille d'bosios. C'est précisément la notion d'impiété, celle aussi de superstition qu'étudie le dernier chapitre de la section. Le contexte historique du dernier tiers du ve siècle est marqué par un intense bouillonnement intellectuel qui remet en cause certaines croyances traditionnelles et qu'Aristophane s'attachera bientôt à discréditer dans les Nuées. Ce climat désécurisant coïncide de surcroît avec un temps de guerre qui attise le besoin de s'assurer la protection des dieux. C'est ce contexte qui permet sans doute de comprendre le vote, à Athènes, en 433-432, d'un décret sur l'asebeia qui fut suivi de plusieurs procès, dont le plus célèbre est assurément celui de Socrate. Peut-être en a-t-on exagéré le nombre et l'importance. L'A. tient en tout cas à rappeler que «le polythéisme grec laisse une grande liberté de fait à la dévotion de chacun, voire même une 'liberté d'incroyance' dans la mesure où elle ne risquait pas de compromettre par ses manifestations la bonne harmonie avec ses dieux » (p. 167). S'il est trace, chez les Grecs, d'une intolérance religieuse caractérisée, c'est plutôt du côté de la législation platonicienne sur l'impiété que promulguent les Lois qu'il convient de se tourner. Quant à la superstition, Platon en trace déjà une ébauche dans les Lois, où il s'en prend à la dévotion débridée de certaines femmes, mais c'est Théophraste, semble-t-il, qui lui donné son nom, deisidaimonia, en brossant, dans ses Caractères, le portrait du superstitieux, celui qui, animé par une crainte irraisonnée des dieux, accumule les actes de dévotion. C'est un thème, on le sait, auquel Plutarque consacrera aussi tout un traité.

Ce résumé trop sec donne du moins une idée du parcours riche et varié qu'effectue cette partie de l'ouvrage dans l'exploration de la notion de piété. On voit combien l'A. a eu le constant souci ne pas traiter la notion de piété de manière abstraite, mais de la situer dans le contexte littéraire où affleurent les mots qui la désignent et dans le contexte historique aussi dans lequel s'inscrivent les œuvres, ne manquant pas de souligner, chaque fois que l'occasion se présente, combien la piété grecque la plus commune est inséparable de préoccupations sociales et politiques. Elle est, oserait-on dire, une vertu citoyenne. Les développements que requiert cette contextualisation contraignent évidemment à faire des choix parmi les auteurs, parmi leurs cuvres comme parmi les thématiques qu'ils abordent relativement au thème. S'il fallait compléter l'éventail, si 
suggestif déjà, qui nous est ici présenté, j'indiquerais pour ma part deux approfondissements souhaitables. Il y aurait lieu tout d'abord, me semble-t-il, d'insister davantage sur la notion de pureté, qui est sans nul doute une composante fréquente et importante de la notion grecque de piété; elle n'est ici que très rarement évoquée. D'autre part, si une approche anthropologique s'impose pour comprendre la conception grecque, il ne serait pas inutile de réfléchir aussi sur l'autre pôle de la relation qu'elle implique, à savoir les dieux et la manière dont les Grecs se les représentent. Sans doute discernerait-on là aussi certaines évolutions et s'apercevrait-on que la piété peut se colorer différemment selon les divinités auxquelles elle s'adresse. Est-ce tout à fait la même piété qui anime les participants aux cortèges carnavalesques de Dionysos, à la procession des Panathénées qui honore Athéna ou encore au pèlerinage qui conduit les mystes à Éleusis et dont Aristophane évoque l'ambiance très fervente dans ses Thesmophories ? Sans doute faudrait-il plus d'un livre pour explorer en ce sens tout le panthéon grec, mais, à défaut d'une telle analyse différentielle, il eût été au moins possible, parallèlement à la longue et pertinente enquête portant les manifestations du culte, d'éclairer aussi la piété des Grecs en disant le divin qu'elle vénère.

La troisième et dernière partie, qui est aussi la plus courte, se propose donc d'analyser «le discours des philosophes sur la piété ». Quatre chapitres jalonnent ce trajet, mais un même fil conducteur les relie, c'est le thème anthropologique de la vie primitive des hommes et de la nature des hommages qu'ils rendaient à leurs dieux. Le principal enjeu, on s'en doute, est le long débat qui va opposer les adeptes du sacrifice sanglant, symbole de la religion des cités, et les partisans d'un retour présumé, tant pour les hommes que pour les dieux, à un régime végétarien qui dispense de faire couler le sang. C'est autour de cette problématique du sacrifice et de l'offrande que serait née la première réflexion sur la piété, liée au développement d'une pensée philosophique, scientifique et aussi politique. Le poète-philosophe Empédocle ouvre la série des penseurs visités; il fait d'Eusébie une figure divine et la montre trônant sur son char et présidant à la Sagesse; il a recours aussi au mot bosiê. Quand, à l'origine, Aphrodite régnait sans partage, prophétise-t-il, il n'y avait ni guerre ni sang versé pour les dieux, lesquels se contentaient de sobres libations. Tel est bien l'âge d'or qu'il convient de restituer. Notre philosophe était, par ces idées, un héritier des Pythagoriciens, mais, contrairement à certaines sectes de la même obédience, il refusait tout compromis visant à tolérer le sacrifice sanglant, remettant ainsi en cause une institution capitale de la cité. Théophraste, qui le cite avec une certaine complaisance, avait écrit un Peri eusebeias dont Porphyre nous a conservé des fragments dans son De abstinentia. Pour définir la meilleure façon d'honorer les dieux, le successeur d'Aristote au Lycée développait dans cet ouvrage toute une théorie historique du sacrifice grec d'où émergeait l'argument décisif de l'ancienneté des sacrifices végétaux; il prônait donc, à tout le moins pour le philosophe, un retour à une alimentation exclusivement végétale, considérant que c'était là une exigence de la vraie piété et que l'abstinence était elle-même une manière d'exercer celle-ci. Sacrifier les êtres inanimés n'est pas permis à ceux qui veulent être vraiment pieux. L'A. analyse finement les longs développements que Théophraste consacrait à ce thème, les comparant aux théories moins engagées que défendait son contemporain Dicéarque, et montrant aussi comment Porphyre tira parti de cet enseignement, en recherchant à son tour un compromis qui ne mette pas le philosophe en contradiction avec la cité : ne peut-on admettre le sacrifice sanglant pour les dieux sans pour autant consommer la chair des victimes sacrifiées ? La chose publique, comme on le voit, restait encore au cour du débat, mais, plutôt qu'une réforme de la cité, la question qui désormais importe, c'est le salut du philosophe. 
Aussi passionnante que soit la lecture de cette dernière partie de l'ouvrage et aussi pertinent que soit le choix de la thématique retenue, l'historien de la philosophie peut difficilement, quand il arrive au terme, réprimer un petit sentiment de frustration. C'est que, tout d'abord, il s'attendait à voir convoqués, au sujet de la piété, des philosophes dont il ne rencontre même pas le nom, ni ici, ni dans les précédents chapitres. À commencer par celui de Xénophane qui, si l'on en croit Aristote (Rbétor. I, 1377a 20), usait déjà des mots eusebès et asebès et qui, en tout cas, s'intéressait vivement à l'attitude pieuse envers la divinité. Ce penseur est bien connu pour sa critique des représentations homériques et hésiodiques des dieux comme aussi de la doctrine de la métempsychose que professait son contemporain Pythagore; on sait aussi qu'il avait jeté les bases d'une théologie nouvelle. Or le fragment 1 donne à penser que ces propos n'étaient pas simplement polémiques ou spéculatifs, mais qu'ils étaient inspirés aussi par une piété sincère. Il recommande à ses concitoyens, en effet, de ne pas perdre de vue la finalité religieuse des banquets festifs qu'ils célèbrent : il faut commencer, dit-il, en chantant la divinité honorée par des mythes pieux (euphêmoi) et des paroles pures (katbaroi), il faut lui offrir des libations avant que de boire et lui demander dans la prière de "pouvoir accomplir les choses justes ". La consigne est aussi, en conséquence, de s'abstenir de célébrer dans des chants les combats des Titans et autres «fictions de jadis » tout comme les guerres civiles. Toujours il faut avoir, au sujet des dieux, « une juste pensée préalable (promêtheiê) ». Ce fragment est d'autant plus remarquable qu'on y voit le philosophe passer d'une notion rituelle de la pureté, appliquée précédemment aux objets et au décor, à celle d'une pureté qui concerne les paroles et la pensée. C'est la première fois aussi qu'un poète ou un penseur grec souligne ainsi l'importance, vitale pour la vie des cités, d'une croyance juste, d'une orthodoxie, pourrait-on dire. Les idées sur la piété de ce philosophe-théologien, très novatrices et très audacieuses pour l'époque, ne resteront pas sans lendemain. Dans la foulée, d'autres penseurs présocratiques, comme Héraclite, mériteraient bien aussi une mention. Mais je voudrais encore attirer simplement l'attention sur la position assez paradoxale que défendit un contemporain de Théophraste et de Dicéarque, le philosophe Epicure, que la piété n'indifférait nullement. Prier, disait-il, est le propre de la sagesse, même s'il n'y a rien à attendre des dieux ni à leur demander. Il ne se contentait pas d'une piété intérieure, mais recommandait à ses disciples de prendre part aux cultes de la cité, en veillant toutefois à ne pas se laisser troubler par les opinions fausses au sujet des dieux. Cette recommandation est d'autant plus remarquable que, dans le même temps, il prônait, comme on sait, un retrait de la vie proprement politique. Voilà donc une autre forme d'bairesis par rapport au système religieux traditionnel; c'est un individualisme qui s'affirme ici, - les vieilles structures de la cité étant désormais ébranlées, - mais qui s'accommode d'un curieux compromis religieux. Toute différente, quelques décennies auparavant, fut la position d'un Aristote et d'un Platon qui entendaient, décidément, rester fidèles au modèle politico-religieux de la cité. J'en veux pour simple preuve le fait qu'au début de son prologue, l'A. cite un texte remarquable des Lois, fait d'une série de recommandations aux citoyens, comme une expression exemplaire de l'idéal grec de piété le plus traditionnel. Mais j'observe en même temps que, si ce philosophe est à nouveau cité fréquemment dans la suite, ce n'est pas précisément pour souligner son effort inlassable pour purifier la piété commune et la moraliser, pour l'intérioriser aussi et la spiritualiser. Je ne ferai pas grief à l'A. d'une telle lacune; son projet initial n'imposait pas pareil détour, d'autant que presque toutes les écoles philosophiques ont nourri de semblables ambitions. Mais, dans une étude qui se veut accueillante à la dimension historique et culturelle, quelques pages supplémentaires consacrées à cette la recherche d'une 
piété plus exigeante et plus personnelle, ou à tout le moins quelques références explicites à des travaux s'intéressant à cet aspect du phénomène religieux en Grèce, auraient été les bienvenues. Car le fait que ce système politico-religieux ait généré, à toutes les époques, des penseurs qui n'ont pas hésité à le remettre en cause avec l'intention de l'amender, le plus souvent de l'intérieur et sans rupture brutale, est sans doute un phénomène culturel majeur, et qui s'est avéré lourd de conséquences pour toute l'histoire de l'Occident.

On aura compris que ces remarques n'entament en rien la valeur du travail réalisé, mais témoignent plutôt du grand intérêt que suscite le thème choisi. Très solidement documentée, - on rappellera que Mme Bruit Zaidmann a déjà signé, avec Mme P. Schmitt Pantel, un ouvrage remarqué sur La religion grecque (Paris, 1991, 3e éd. 1999) - méthodiquement conduite, d'une présentation très soignée, écrite aussi dans un style sobre et élégant, l'étude se termine par un "épilogue » très dense (p. 211-217); elle est accompagnée d'instruments précieux : une bibliographie de quelques 200 titres, une table des illustrations ainsi qu'un index général reprenant les noms propres anciens, les realia et les principales notions relatives au thème traité. Voilà donc un ouvrage qui, sur un sujet difficile, apporte une contribution originale et importante à notre connaissance de la religion grecque.

Université de Liège

Département de Philosophie

7, place du 20-Août,

$\mathrm{B}-4000$ LIÈGE

andre.motte@ulg.ac.be

\section{Comptes rendus et notices bibliographiques}

Yves Lehmann (dir.), Dominique Briquel, Gérard Freyburger, Mireille Hadas-lebel, Vinciane Pirenne-Delforge, Charles Marie Ternes, Religions de l'Antiquité, Paris, P.U.F., 1999. 1 vol, $15 \times 21,5 \mathrm{~cm}, \mathrm{xIV}+592$ p., 34 fig, (Collection Premier Cycle). ISBN : 2-13-048221-X.

Présenter en un seul ouvrage, à l'intention des étudiants du premier cycle des études universitaires, les principales religions qui se sont développées autour du bassin méditerranéen est assurément une excellente idée. C'est une manière, tout d'abord, de faire voir la grande diversité et l'importance des courants religieux qui ont imprégné les cultures concernées. C'est une façon aussi d'encourager les comparaisons et de contribuer à mettre en relief les caractères propres à chaque religion. L'entreprise n'en était pas moins une gageure, car réaliser pareilles synthèses, avec toutes les garanties de sérieux, n'est pas à la portée du premier venu et ne peut être d'ailleurs le fait d'un seul homme. Elle nécessitait la collaboration de spécialistes avertis, œuvrant dans une optique scientifique commune, mais disposant d'une latitude suffisante pour adapter leur étude aux particularités de la matière; il y fallait aussi de solides qualités pédagogiques. On peut dire que, sous ces différents rapports, l'ouvrage est globalement une réussite.

Ses sept parties concernent successivement «la religion étrusque », p. 7-75 (D. Briquel), « la religion grecque ", p. 79-175 (V. Pirenne-Delforge), « la religion romaine traditionnelle ", p. 179-246 (Y. Lehmann), "les religions à mystères dans 